\title{
AVALIAÇÃO DO INÍCIO DE OPERAÇÃO DAS PRENSAS DE ROLOS DO PROJETO MINAS-RIO*
}

\author{
Douglas Batista Mazzinghy ${ }^{1}$ \\ José Francisco Cabello Russo ${ }^{1}$ \\ Fabrício Maia $^{2}$ \\ Mauro Lúcio da Costa ${ }^{2}$ \\ Antônio Eduardo Pereira ${ }^{2}$ \\ Ricardo Oliveira Coutinho ${ }^{2}$ \\ Rogério Moreira Alves ${ }^{2}$ \\ Claudio Luiz Schneider ${ }^{3}$
}

\section{Resumo}

O projeto Minas-Rio iniciou as suas operações no final de 2014 e se encontra na fase de ramp-up buscando a capacidade nominal. O circuito de cominuição inclui três prensas de rolos em circuito aberto com o objetivo de criar micro fissuras no minério e assim reduzir o consumo energético da etapa seguinte, o circuito de moagem de bolas. Este tipo de fluxograma, com prensa de rolos (circuito aberto) seguido de moagem de bolas (circuito fechado), é conhecido como circuito híbrido. A literatura apresenta este circuito híbrido como o de maior eficiência energética. $A$ maior preocupação com relação as prensas de rolos aplicada aos minérios de ferro é a geração excessiva de finos que poderiam elevar o perda de material na etapa de deslamagem, anterior a etapa de concentração por flotação. Os resultados obtidos através de amostragem das prensas mostraram que não houve geração excessiva de finos. Neste trabalho são apresentados os resultados iniciais da operação das prensas de rolos.

Palavras-chave: HPGR; Prensa de rolos; Minas-Rio; Minério de ferro; Circuito híbrido.

\section{Abstract \\ MINAS-RIO PROJECT START-UP PERFORMANCE EVALUATION}

The Minas-Rio project began operations in late 2014 and is in the ramp-up phase seeking nominal capacity. The comminution circuit includes three HPGR's in open circuit in order to create micro-cracks in the ore and thus reduce energy consumption in the next step, the grinding balls circuit. This configuration with HPGR (open circuit) followed by grinding balls (closed circuit) is known as a hybrid circuit. The literature presents this hybrid circuit as high energy efficient. The biggest concern about the HPGR's applied to iron ore is the excessive generation of fines that could raise the loss of material in desliming stage, before the concentration step by flotation. This paper presents the initial results of the HPGR's performance.

Keywords: HPGR; Minas-Rio; Iron ore; Hybrid circuit.

1 Desenvolvimento de Processos, Anglo American Minério de Ferro Brasil, Conceição do Mato Dentro, MG, Brasil.

2 Operação, Anglo American Minério de Ferro Brasil, Conceição do Mato Dentro, MG, Brasil.

3 Pesquisador Sênior, Centro de Tecnologia Mineral, Rio de Janeiro, RJ, Brasil. 


\section{INTRODUÇÃO}

\section{$1.1 \quad$ Minas-Rio}

O Projeto Minas-Rio, de propriedade da Anglo American, iniciou as suas operações no final de 2014. O circuito de britagem é dividido em etapas de britagem primária composta por grelhas vibratórias e por britadores de mandíbulas e britagem secundária composta por peneiras do tipo banana e por britadores hidrocônicos em circuito fechado. A etapa de moagem é dividida em duas etapas, a primeira etapa com prensas de rolos em circuito aberto e a segunda etapa por moinhos de bolas em circuito fechado com hidrociclones. A Figura 1 mostra o fluxograma da planta de processamento mineral do Minas-Rio.

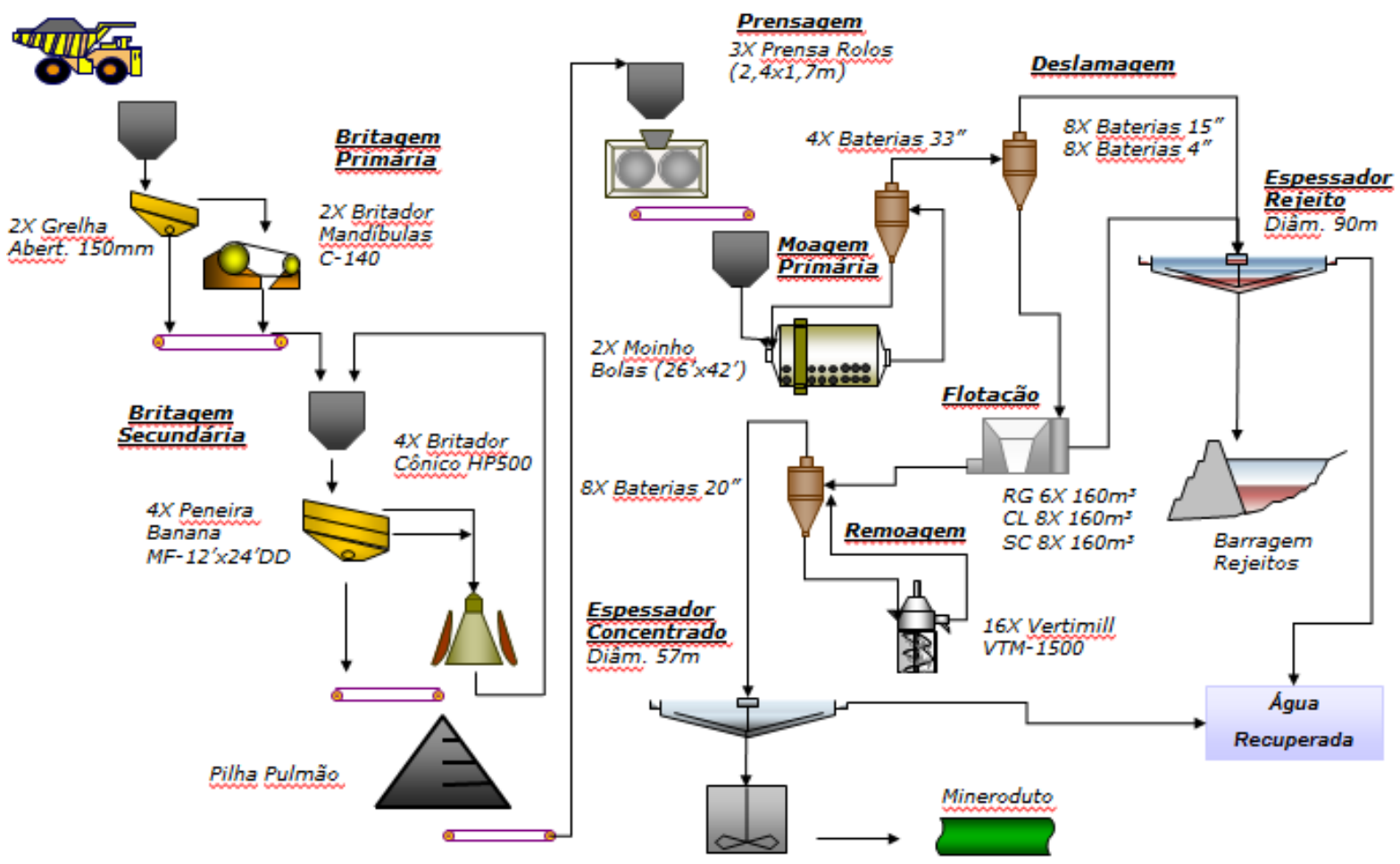

Figura 1. Fluxograma da planta de processamento mineral do Minas-Rio.

\subsection{Prensa de Rolos (HPGR)}

As prensas de rolos ou HPGR (High Pressure Grinding Rolls) são equipamentos relativamente novos na indústria mineral. $O$ conceito da prensa de rolos foi proposto por Klaus Schoenert [4] na Alemanha. A primeira aplicação industrial ocorreu em 1985 na indústria de cimento [2] e a primeira aplicação para metais básicos ocorreu em Cerro-Verde no Peru [5].

A Figura 2 apresenta o conceito de uma prensa de rolos. O material é alimentado entre os rolos que giram em sentidos oposto de forma a conduzir o material alimentado para o vão entre os rolos, chamado de gap. O sistema hidráulico tem como objetivo aplicar pressão de forma a moer o material entre os rolos. Um sistema com cilindros de hidrogênio tem a função de amortecer a variação de pressão exercida pelos materiais de diferentes competências alimentados na prensa de rolos. 


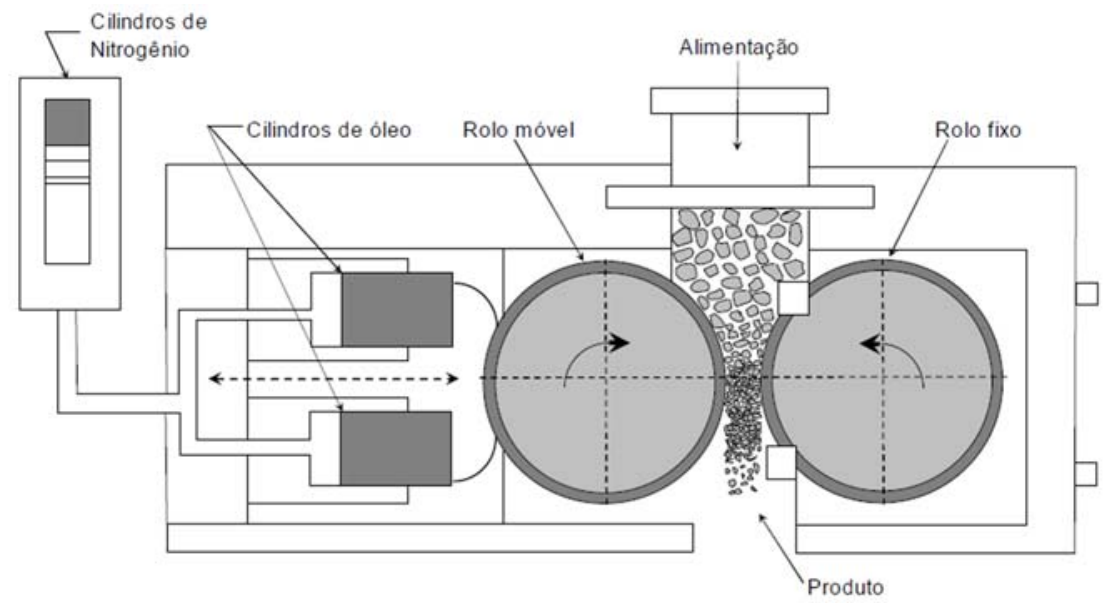

Figura 2. Conceito prensa de rolos.

A Figura 3 mostra as prensa de rolos do sistema Minas-Rio fornecidas pela Polysius (atualmente ThyssenKrupp).

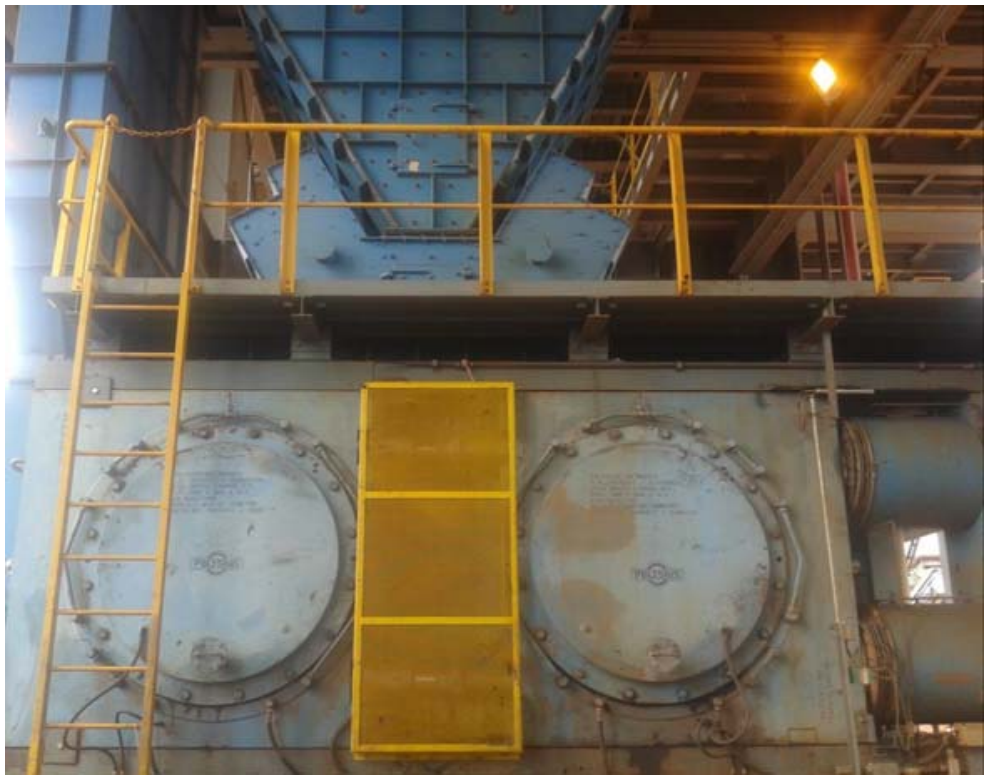

Figura 3. Prensa de Rolos do sistema Minas-Rio.

O Minas-Rio possui três prensas de rolos de 2,40 $\mathrm{m}$ de diâmetro por 1,65 $\mathrm{m}$ de largura. Cada prensa de rolos possui 2 motores de $2400 \mathrm{~kW}$ totalizando $4800 \mathrm{~kW}$ de potência por prensa de rolos.

\subsection{Circuito Híbrido}

O circuito híbrido de moagem consiste em uma prensa de rolos em circuito aberto seguida de um circuito de moinho de bolas fechado com classificador. A Figura 4 apresenta o fluxograma do circuito híbrido de moagem. 


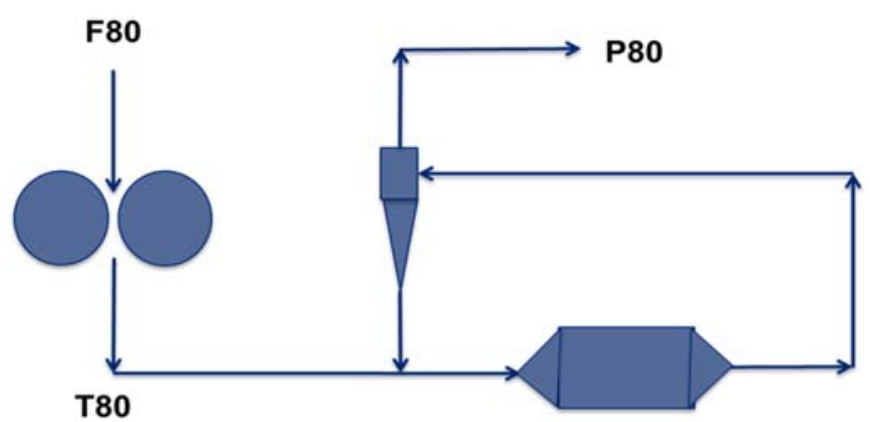

Figura 4. Conceito do circuito híbrido de moagem

Segundo Fuesternau et al. [1] o circuito híbrido de moagem é o que apresenta a maior eficiência energética.

A Figura 5 mostra os diagramas de taxa de redução por energia aplicada para a prensa de rolos, para o moinho de bolas e para a combinação destes dois equipamentos.

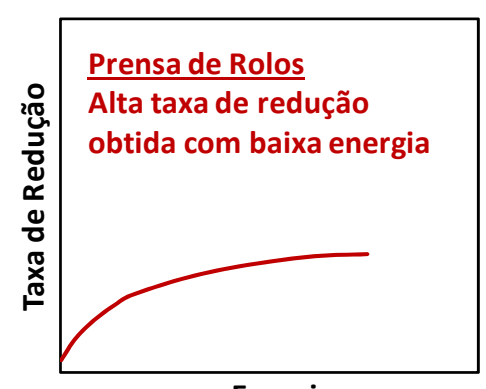

Energia

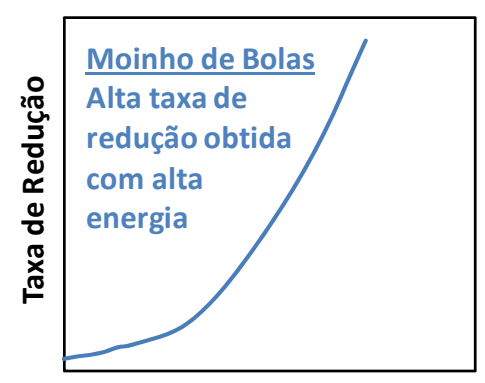

Energia

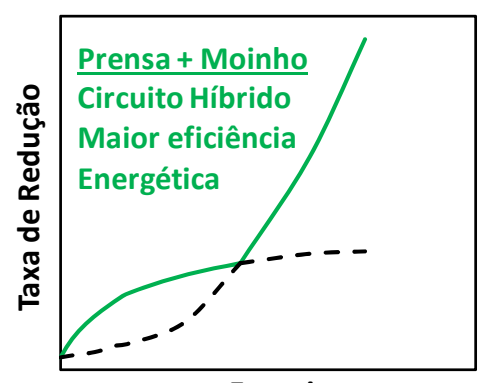

Energia

Figura 5. Diagramas de taxa de redução por consumo energético.

A prensa de rolos consegue altas taxas de redução com reduzida aplicação de energia. Após a aplicação de uma quantidade maior de energia a taxa de redução da prensa de rolos é quase constante (crescimento não significativo).

O moinho de bolas somente consegue altas taxas de redução com elevada aplicação de energia.

O circuito híbrido de moagem combina o que há de melhor da prensa de rolos e do moinho de bolas. Existe uma partição de energia ideal entre a prensa de rolos e o moinho de bolas onde se obtém o menor consumo energético.

\subsection{Objetivo}

O objetivo deste trabalho é apresentar e discutir os resultados obtidos através de amostragem das prensas de rolos do projeto Minas-Rio. A geração excessiva de finos é a principal informação investigada neste trabalho.

\section{MATERIAIS E MÉTODOS}

No total foram realizadas quatro amostragens na planta industrial em diferentes condições operacionais. Os fluxos de alimentação e produto da prensa de rolos foram amostrados manualmente e para cada amostra foi determinada a distribuição 
granulométrica, inclusive a fração fina (ciclosizer), densidades real e aparente e umidade.

Os dados das variáveis de processo (taxa de alimentação, pressão, potência, abertura entre os rolos) foram coletados através do sistema de controle, quando disponível.

\section{RESULTADOS E DISCUSSÃO}

As amostragens foram realizadas em dias distintos e em condições operacionais diferentes.

As amostragens 1 e 2 foram realizadas com diferentes litologias de minérios e com abertura entre rolos (gap) de $57 \mathrm{~mm}$.

As amostragens 3 e 4 foram realizadas com a mesma litologia variando a abertura entre os rolos (gap) de $57 \mathrm{~mm}$ e $49 \mathrm{~mm}$.

Em todas as amostragens a pressão de trabalho registrada estava em aproximadamente 70 bar.

A Tabela 1 apresenta a caracterização das amostras das amostragens 1 e 2.

Tabela 1 - Caracterização da amostragem 1 e 2

\begin{tabular}{|c|c|c|c|c|}
\hline \multirow{2}{*}{ HPGR } & \multicolumn{2}{|c|}{ Amostragem 1} & \multicolumn{2}{|c|}{ Amostragem 2} \\
\hline & Alim. & Prod. & Alim. & Prod. \\
\hline Umidade (\%) & \multicolumn{2}{|c|}{4,1} & \multicolumn{2}{|c|}{4,6} \\
\hline Densidade (t/m3) & \multicolumn{2}{|c|}{3,6} & \multicolumn{2}{|c|}{3,7} \\
\hline Dens. Apar. (t/m3) & \multicolumn{2}{|c|}{2,3} & \multicolumn{2}{|c|}{2,2} \\
\hline d98 $(\mu \mathrm{m})$ & 26668 & 18126 & 18161 & 10930 \\
\hline $\mathrm{d} 80(\mu \mathrm{m})$ & 4223 & 3418 & 773 & 334 \\
\hline $\mathrm{d} 50(\mu \mathrm{m})$ & 135 & 112 & 90 & 73 \\
\hline $\mathrm{d} 20(\mu \mathrm{m})$ & 35 & 30 & 33 & 29 \\
\hline
\end{tabular}

A Figura 6 apresenta as curvas granulométricas de alimentação e produto das prensas das amostragens 1 e 2.

Não houver formação de flake (material prensado) no produto. A densidade aparente do produto foi a mesma da alimentação da prensa. 


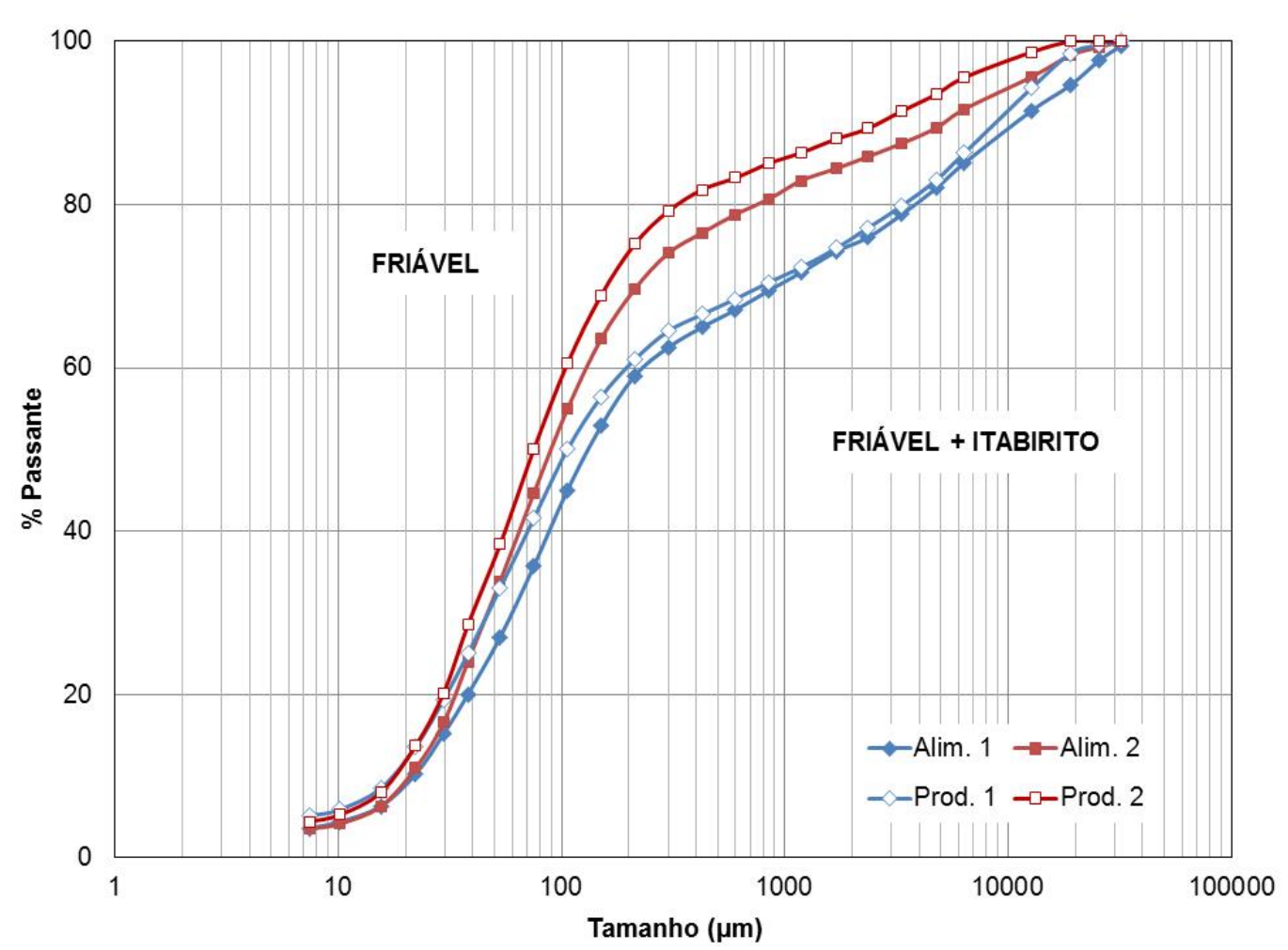

Figura 6. Curvas granulométricas obtidas através das amostragens 1 e 2 .

Estudos anteriores [3] mostraram que as duas litologias principais do Minas-Rio, chamadas de Friável e Itabitito, possuem comportamentos distintos na moagem. A litologia Friável possui distribuição granulométrica com grande presença de finos e maior consumo energético. A litologia Itabirito possui comportamento oposto, menor presença de finos e menor consumo energético. Uma investigação utilizando análise de imagem (MLA - Mineral Liberation Analyser) foi conduzida e identificou que os grãos de quartzo da litologia Friável são maiores, justificando assim o maior consumo energético desta litologia, mesmo com distribuição granulométrica com maior presença de finos.

Não foi possível registrar os dados de potência das prensas de rolos nas amostragens 1 e 2, não sendo possível verificar/comprovar este comportamento.

A Tabela 2 apresenta a caracterização das amostragens 3 e 4.

Tabela 2 - Caracterização da amostragem 3 e 4

\begin{tabular}{|c|c|c|c|c|}
\hline \multirow{2}{*}{ HPGR } & \multicolumn{2}{|c|}{ Amostragem 3} & \multicolumn{2}{|c|}{ Amostragem 4} \\
\hline & Alim. & Prod. & Alim. & Prod. \\
\hline Umidade (\%) & \multicolumn{2}{|c|}{4,4} & \multicolumn{2}{|c|}{7,1} \\
\hline Densidade (t/m3) & \multicolumn{2}{|c|}{3,8} & \multicolumn{2}{|c|}{3,9} \\
\hline Dens. Apar. (t/m3) & \multicolumn{2}{|c|}{2,6} & \multicolumn{2}{|c|}{2,8} \\
\hline d98 $(\mu \mathrm{m})$ & 20209 & 18718 & 25010 & 11952 \\
\hline $\mathrm{d} 80(\mu \mathrm{m})$ & 2905 & 1401 & 4301 & 654 \\
\hline $\mathrm{d} 50(\mu \mathrm{m})$ & 125 & 85 & 130 & 73 \\
\hline $\mathrm{d} 20(\mu \mathrm{m})$ & 38 & 33 & 36 & 31 \\
\hline
\end{tabular}

A Figura 7 apresenta as curvas granulométricas de alimentação e produto das prensas das amostragens $3(g a p=57 \mathrm{~mm})$ e $4($ gap $=49 \mathrm{~mm})$. 


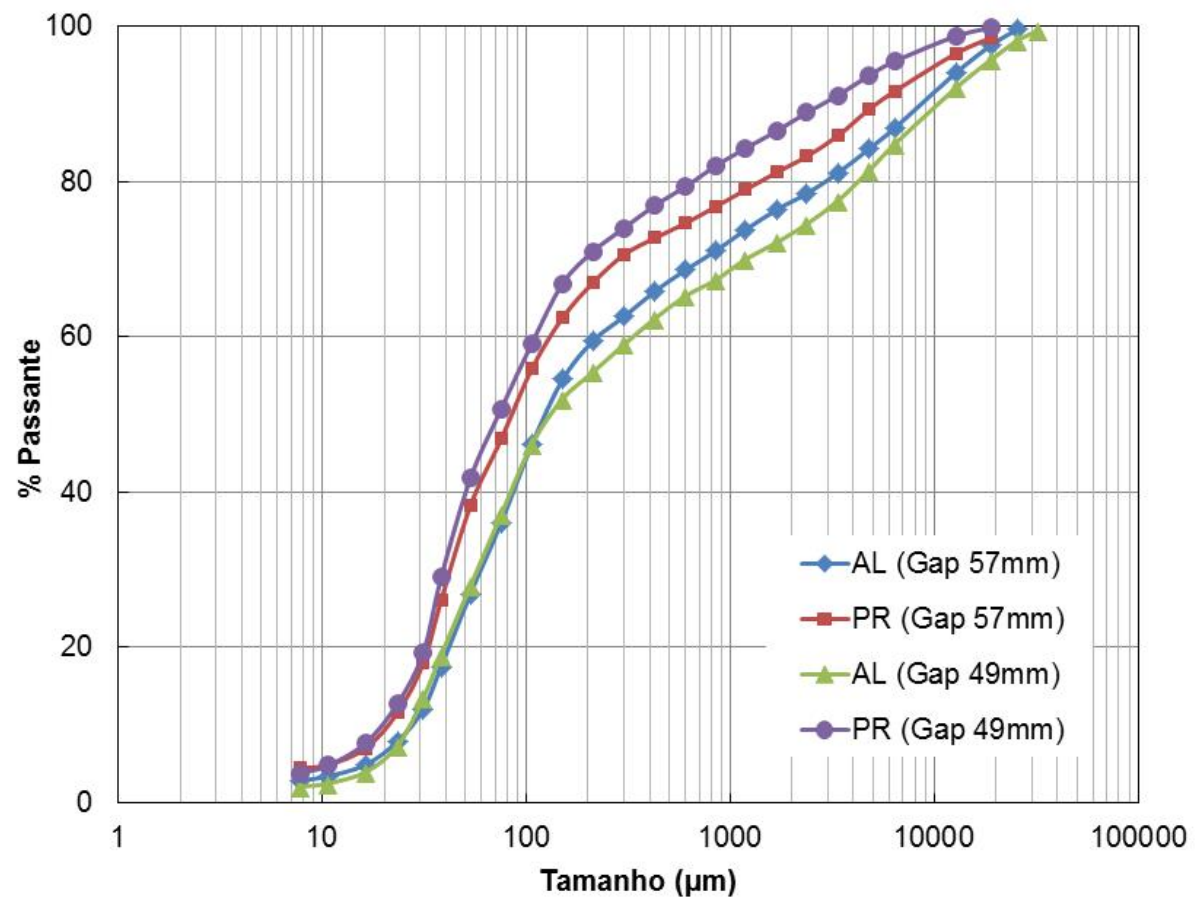

Figura 7. Curvas granulométricas obtidas através das amostragens 3 e 4 .

Novamente não houver formação de flake (material prensado) no produto. A densidade aparente do produto foi a mesma da alimentação da prensa.

Com um gap reduzido $(49 \mathrm{~mm})$ a taxa de redução foi maior nas frações grossas e praticamente não se alterou nas frações finas, quando comparada com o gap maior (57 mm).

Durante as amostragens 3 e 4 os dados de taxa de alimentação (t/h) e potencia $(\mathrm{kW})$ foram registrados. A amostragem $3(\mathrm{gap}=57 \mathrm{~mm}$ ) apresentou consumo específico de $1,01 \mathrm{kWh} / \mathrm{t}$ e a amostragem $4(\mathrm{gap}=49 \mathrm{~mm})$ apresentou consumo específico de $1,53 \mathrm{kWh} / \mathrm{t}$.

A maior taxa de redução da amostragem 4 foi obtida com maior consumo energético.

A energia específica disponível no Minas-Rio é de 2,22 kWh/t, conforme mostrado no cálculo a seguir:

\section{3 prensas * (cada prensa possui 2 motores de $2400 \mathrm{~kW}$ ) $=14400 \mathrm{~kW}$ (total) $14400 \mathrm{~kW}$ (total) / $6492 \mathrm{t} / \mathrm{h}$ (nominal) = 2,22 kWh/t}

Pode se afirmar que é possível otimizar as prensas de rolos considerando menores aberturas entre rolos (gap), o que implica em maior energia demandada e maior redução do material processado.

\section{CONCLUSÃO}

As prensas de rolos do projeto Minas-Rio foram amostradas e os resultados obtidos foram avaliados com relação a possível geração excessiva de finos, o que poderia prejudicar a etapa seguinte de deslamagem. Contudo, na condição atual de operação das prensas de rolos (ramp up) não houve geração excessiva de finos que pudesse prejudicar a etapa de deslamagem.

Através dos dados obtidos é possível afirmar que existe a possibilidade de otimização do processo de moagem (prensa de rolos + moinho de bolas) escolhendo 
a partição ideal de energia entre as prensas de rolos e os moinhos de bolas de forma a obter a maior taxa de redução com o menor consumo energético.

Os próximos passos são: continuar com as amostragens para identificar a partição ideal de energia entre as prensas de rolos e os moinhos de bolas e verificar as frações finas das curvas granulométricas com o objetivo de monitorar uma possível geração excessiva de finos.

\section{Agradecimentos}

Agradecimento especial a Anglo American por autorizar a divulgação dos dados e promover assim a divulgação do uso de novas tecnologias aplicadas aos projetos minério de ferro.

\section{REFERÊNCIAS}

1 Fuerstenau, D.W. \& Vazquez-Favela. On assessing and enhancing the energy efficiency of comminution process, Minerals and Metallurgical Processing, 1997.

2 Kellerwessel, $\mathrm{H}$. High pressure material bed comminution in practice, Zement-Kalk-Gips, 1990, (2), pp.57-64.

3 Mazzinghy, D.B.; Turrer, H.D.G.; Russo, J.F.C; Tavares, L.M. Influence of the mineralogical structure in grinding specific energy consumption of itabirite iron ores. In: Anais do Comminution 2014, Cape Town, África do Sul, 2014, 9p.

4 Schonert, K. "Verfahren zur Fein- und Feinstzerkleinerung von Materialien sproden Stoffverhaltens," German Patent 2708 053, 1979.

5 Vanderbeek, J.L, Linde, T.B., Brack, W.S., Marsden, J.O. HPGR implementation at Cerro Verde, in SAG 2006 Conference, Reprints, CIM, Vancouver, BC, Canada, 2006., 19 pp. 